\title{
Molecule-based magnets
}

\author{
J V YAKHMI \\ Bhabha Atomic Research Centre, Mumbai 400 085, India
}

\begin{abstract}
The conventional magnetic materials used in current technology, such as, $\mathrm{Fe}_{2} \mathrm{Fe}_{2} \mathrm{O}_{3}, \mathrm{Cr}_{2} \mathrm{O}_{3}$, $\mathrm{SmCo}_{5}, \mathrm{Nd}_{2} \mathrm{Fe}_{14} \mathrm{~B}$ etc are all atom-based, and their preparation/processing require high temperature routes. Employing self-assembly methods, it is possible to engineer a bulk molecular material with long-range magnetic order, mainly because one can play with the weak intermolecular interactions. Since the first successful synthesis of molecular magnets in 1986, a large variety of them have been synthesized, which can be categorized on the basis of the chemical nature of the magnetic units involved: organic-, metal-based systems, heterobimetallic assemblies, or mixed organic-inorganic systems. The design of molecule-based magnets has also been extended to the design of poly-functional molecular magnets, such as those exhibiting second-order optical nonlinearity, liquid crystallinity, or chirality simultaneously with long-range magnetic order. Solubility, low density and biocompatibility are attractive features of molecular magnets. Being weakly coloured, unlike their opaque classical magnet 'cousins' listed above, possibilities of photomagnetic switching exist. Persistent efforts also continue to design the ever-elusive polymer magnets towards applications in industry. While providing a brief overview of the field of molecular magnetism, this article highlights some recent developments in it, with emphasis on a few studies from the author's own lab.
\end{abstract}

Keywords. Molecular lattices; spin-spin interaction; photo-induced magnetism; single molecule magnets.

\section{Introduction}

Most molecular materials are organic in nature. Molecular crystals are made up of well-defined molecules, which do not change their geometries appreciably upon entering the crystal lattice. This is because intermolecular interactions are non-covalent in nature, viz. hydrogen bonding, Van der Waals interactions, donor-acceptor charge transfer, etc that are much weaker than the energies of typical chemical bonds, ionic or covalent. This provides an interesting possibility to modify the properties of a molecular solid in a predetermined way by attaching a function to the building-block (i.e. the molecule), and thus engineer a bulk molecular material with designer characteristics. Chemical synthesis, at ambient temperature, provides immense flexibility towards the synthesis of new molecular materials designed to perform several functions originally attributed to the metallic lattices, such as, high electric conductivity or photoactivity in polymers, and superconductivity in charge-transfer organic complexes/ fullerenes. Quite obviously, there has been a strong urge to develop ferro(ferri)magnetic molecular materials, too, by designing new combinations of interactions between magnetic centres in organic (or polymeric) materials, preferably with $p$-orbital based spins. Theoretical models did predict the possibility of attaining long-range magnetic order in molecular materials. However, organic

(yakhmi@barc.gov.in) compounds are mostly diamagnetic, with closed-shell structures, and even if one (or more) unpaired electron is maintained stably per organic molecule, stabilization of a triplet state (parallel alignment of spins) requires that the orthogonality conditions are satisfied (Hund's rules), which is difficult, so they prefer to get antiferromagnetically coupled with no spontaneous magnetic moment. No wonder, the conventional magnetic materials used in present-day technology, such as, $\mathrm{Fe}, \mathrm{Fe}_{2} \mathrm{O}_{3}, \mathrm{Cr}_{2} \mathrm{O}_{3}, \mathrm{SmCo}_{5}$, $\mathrm{Nd}_{2} \mathrm{Fe}_{14} \mathrm{~B}$, etc are all atom-based materials, in which magnetic order arises from co-operative spin-spin interactions between unpaired electrons located in $d$-orbitals or f-orbitals. But their synthesis typically depends on solid-state chemistry or high temperature metallurgical routes.

Typical synthetic approach to design molecule-based magnets consists of choosing molecular precursors, each bearing a unpaired spin (the function, as shown in figure 1), and assembling them in such a way that there is no compensation of spins at the scale of the crystal lattice (Kahn 1993; Itoh and Kinoshita 2000; Miller 2000). The design of molecular magnets has also employed the tools of supramolecular chemistry (Kahn 1996). Magnetism being a co-operative effect, the spin-spin interaction must extend to all the three dimensions, either through space or through bond. The interactions between spin carriers may occur through space, in which case we have a genuine molecular lattice, or, through bond when we are faced with a polymeric or extended structure. In the latter case, the interactions are usually much stronger, 
particularly so when the bridging ligands are conjugated. The design of a molecular magnet requires that: (a) all the molecules in the lattice have unpaired electrons; and (b) the unpaired electrons should have their spins aligned parallel along a given direction (Yakhmi 2003).

\section{Purely organic magnets}

The first two ferro(ferri)magnetic molecular compounds exhibiting a spontaneous magnetization below a certain temperature, $T_{\mathrm{c}}$, were reported in 1986 (Miller et al 1986; Pei et al 1986), and subsequently molecular magnets of many different categories have been synthesized and interest in the field of molecule-based magnets has been growing steadily. The discovery of ferromagnetism involving $p$-electrons in iron-containing organic-based material (Miller et al 1986) was an important step forward because magnetism in metal-free compounds must involve electrons from $p$-orbitals, which was considered impossible not long ago. Despite a significant progress made in the preparation of $\pi$-conjugated oligomers and polymers with large values of spin quantum number, $S$, persistent efforts to design polymer magnets have not borne fruit yet, though success in this endeavour, if and when achieved, would have a huge impact on applications in industry (Ovchinnikov 1978; Itoh and Kinoshita 2000; Rajca et al 2001). Multi-orbital ferromagnets such as $\mathrm{TDAE}-\mathrm{C}_{60}$ (Allemand et al 1991) are known, but

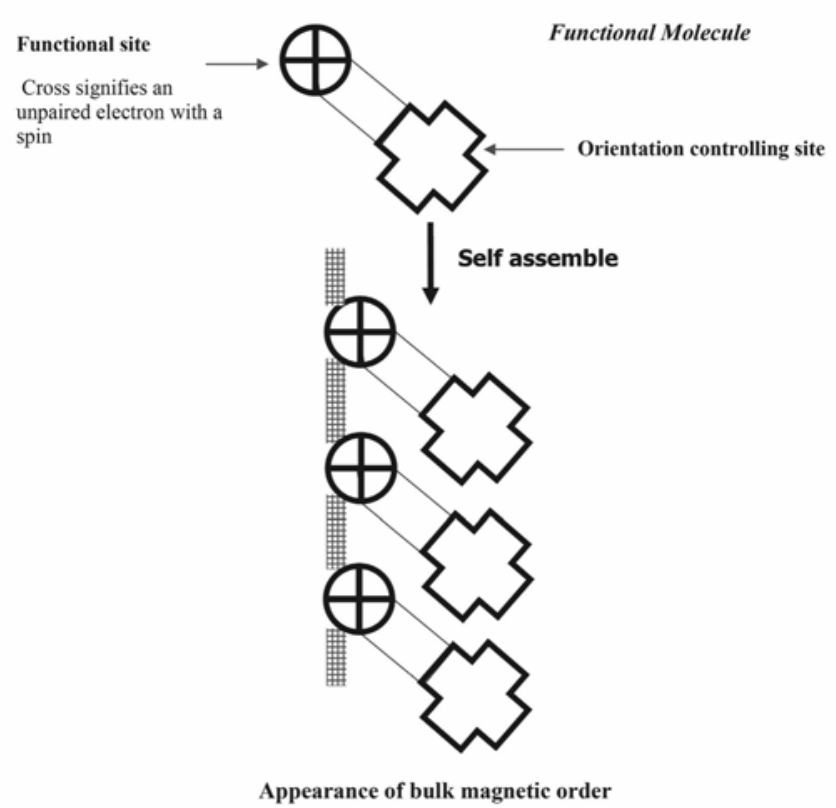

Figure 1. Cartoon depicting the assembly of a molecule-based magnet from functional molecules (precursors), each bearing an unpaired spin (the function), in such a way that there is no compensation of spins at the scale of the crystal lattice, yielding a three-dimensional lattice with a long-range magnetic order. organics exhibiting (single-)band ferromagnetism have yet to be synthesized. Spin density functional calculations (Arita et al 2002) have proposed the possibility of band ferromagnetism in purely organic polymeric structures like PAT [poly(4-amino 1,2,4 triazole], which are chains of five-membered rings. They predict that when the flat band of such materials is made half-filled, with appropriate dopings using, say, a field-effect transistor structure, the ground state will become ferromagnetic.

Magnetism being a co-operative effect, the spin-spin interaction must extend to all the three dimensions. Specific occurrence of spin delocalization and spin polarization in molecular lattices, unlike in the case of ionic/ metallic compounds, is helpful in bringing about ferromagnetic interaction by facilitating necessary intermolecular exchange interactions. The delocalization of spin density in certain molecules makes it possible for magnetic interactions to take place across extended bridges between magnetic centres far apart from each other, propagating through conjugated bond linkages, which act as molecular wires. Spin polarization, i.e. the simultaneous existence of positive and negative spin densities at different locations within a given radical is crucial for intermolecular exchange interactions to bring about ferromagnetic interaction between organic radicals, as per McConnell's (1963) model. Spin density across different regions of the nitronyl nitroxide radical NITR $(\mathrm{R}=$ alkyl), a versatile building block with spin $S=1 / 2$ ground state (figure 2), for instance, shows positive values, equally delocalized between $\mathrm{N}$ and $\mathrm{O}$ within each $\mathrm{N}-\mathrm{O}$ group, and a small negative value on the bridging $s p^{2}$ carbon, due to spin polarization. By substituting different alkyl groups (like $\mathrm{R}=$ benzyl, isopropyl, methyl, ethyl, phenyl, etc) in NITR, one can tune the single-radical ground state to establish new exchange pathways through varied coordination sites. For instance, ferromagnetism at $0.6 \mathrm{~K}$ arises solely from $p$-orbital spins in the $\beta$-phase of $\mathrm{R}=$ phenyl compound (4-nitrophenylnitronyl nitroxide) ( $p$-NPNN, with formula $\mathrm{C}_{13} \mathrm{H}_{16} \mathrm{~N}_{3} \mathrm{O}_{4}$, shown in figure 2), a metal-free organic magnet which contains only $\mathrm{C}, \mathrm{H}, \mathrm{N}$, and O elements (Tamura et al 1991).

Ferromagnetism has also been obtained for the purely organic fullerene-based charge-transfer material, [tetrakis

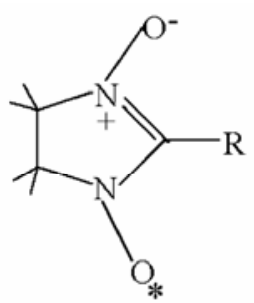

(A)

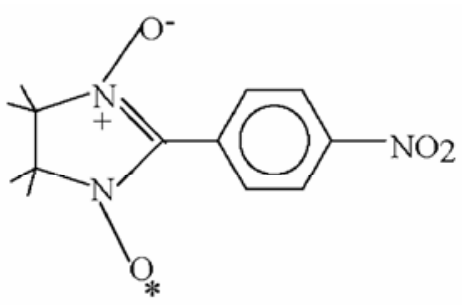

(B)
Figure 2. The chemical structure of (A) NITR and (B) $p$-NPNN (* indicates an unpaired electron). 
(dimethylamino)ethylene $]\left[\mathrm{C}_{60}\right]$ with $T_{\mathrm{c}}$ of $16 \cdot 1 \mathrm{~K}$ (Allemand et al 1991). Fullerene has no intrinsic magnetic moment. For a magnetic moment to exist, an electron must be transferred to $\mathrm{C}_{60}$ from a donor molecule. Another example of a fullerene-based ferromagnet was the cobaltocene-doped derivative, which has a $T_{\mathrm{c}}$ of $19 \mathrm{~K}$ (Mrzel et al 1998). The highest ordering temperature reported to date for an organic magnet has been for the $\beta$-phase of the 4'-cyanotetrafluorophenyldithiadiazolyl, a sulfur-based free radical, which was found to be a weak ferromagnet below 35.5 K (Banister et al 1996; Palacio et al 1997). Under a pressure of 16 kilobars, this temperature can be raised to $65 \mathrm{~K}$ (Mito et al 2001).

\section{Ferrimagnetic building-blocks}

A powerful strategy, pioneered by $\mathrm{O}$ Kahn, to build a molecule-based magnet is based on the use of ferrimagnetic chains containing alternating spins of unequal magnitude, $S_{\mathrm{A}} \neq S_{\mathrm{B}}$, and assembling them in such a way that there is a net spin, leading to a long-range magnetic order in the lattice (Kahn 1993, 1995). Here, $S_{\mathrm{A}}$ stands for the large spin and $S_{\mathrm{B}}$ for the small spin on two different spin carriers, $\mathrm{A}$ and $\mathrm{B}$, such as $\mathrm{Mn}^{\mathrm{II}}$ ions $(S=5 / 2)$ and $\mathrm{Cu}^{\mathrm{II}}$ ions $(S=1 / 2)$, respectively, within the same molecular precursor (figure 3). A large number of molecular (ferro)ferrimagnets have been assembled using this technique, the spin carriers in them being either two different metal ions (Nakatani et al 1989; Okawa et al 1994) or a metal ion and an organic radical (Caneschi et al 1989; Broderick et al 1990; Inoue and Iwamura 1994; Inoue et al 1996), with intervening ligands which serve as effective exchange pathways.

Heterobimetallic species, in which two different metal ions are bridged by extended bisbidentate ligands such as oxamato (Kahn et al 1988; Stumpf et al 1993a), oxamido (Nakatani et al 1989; Pei et al 1991), or oxalato (Okawa et al 1994), in particular, allow a variety of spin topologies.
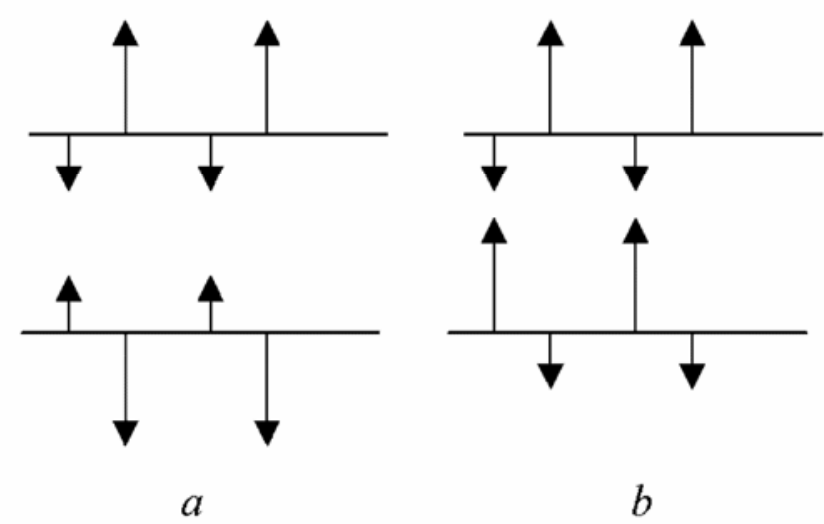

Figure 3. Assembly of ferrimagnetic chains leading to a net a zero, or b. non-zero magnetic moment in bulk.
Using this principle, Kahn's group synthesized several $\mathrm{Mn}(\mathrm{II}) \mathrm{Cu}(\mathrm{II})$ molecular magnets in which the ferrimagnetic interactions are propagated through bisbidentate ligands, viz. $\mathrm{MnCu}(o p b a) \cdot 0 \cdot 7 \mathrm{DMSO}$ which is synthesized by reacting the $\mathrm{Cu}(\mathrm{II})$ precursor $\mathrm{Cu}(o p b a)^{2-}$ (figure 4), where opba stands for ortho-phenylenebis(oxamato), with a divalent ion, $\mathrm{Mn}$ (II) in a 1:1 stoichiometry. This compound is an amorphous magnet with a spontaneous magnetization below $T_{\mathrm{c}}=6.5 \mathrm{~K}$. Aiming at increasing the Curie temperatures of this class of compounds, they synthesized $2: 3 \mathrm{Mn}(\mathrm{II}) \mathrm{Cu}(\mathrm{II})$ compounds $\mathrm{A}_{2} \mathrm{M}_{2}[\mathrm{Cu}(o p b a)]_{3} \cdot n$ solv with two-dimensional character, by employing $[\mathrm{Cu}(o p b a)]^{2-}$ to cross-link the chains in a two-dimensional network, in the presence of two equivalents of the cation $\mathrm{A}^{+}$. Among them is $\left(\mathrm{NBu}_{4}\right)_{2} \mathrm{Mn}_{2}[\mathrm{Cu}(o p b a)]_{3}$. $6 \mathrm{DMSO} \cdot 1 \mathrm{H}_{2} \mathrm{O}$, exhibiting a transition at $T_{\mathrm{C}}=15 \mathrm{~K}$ towards a ferromagnetically ordered state (Stumpf et al $1993 \mathrm{a}$ ), the $T_{\mathrm{C}}$ value of which rises to $22.5 \mathrm{~K}$ when all the solvent molecules are removed (Stumpf et al 1993a; Chavan et al 1996). Elucidation of structure of $1: 2$ $\mathrm{Mn}(\mathrm{II}) \mathrm{Cu}(\mathrm{II})$ compound $\left(\mathrm{NBu}_{4}\right)_{2} \mathrm{Mn}[\mathrm{Cu}(o p b a)]_{2}$ by us revealed a new crystallographic arrangement among the class of ' $o p b a$ ' molecular magnets (Neels et al 2001), which were known till then to exist as one-dimensional chains when the ratio is $1: 1$, or as planar graphite-like sheets for the $2: 3$ ratio.

An effective process for assembling spin-bearing precursors is when polymerization is associated with dehydration. When the precursors are linked to each other after polymerization, the interaction involving a spin carrier A from a given unit and a spin carrier B from the adjacent unit becomes large, and the resulting ground state spin of the polymer $[\mathrm{AB}]_{n}$, where $n$ is the number of units, becomes $n\left(S_{\mathrm{A}}-S_{\mathrm{B}}\right)$. If $n$ is infinite and the system is three-dimensional, a long-range ferrimagnetic ordering should occur, which can also be considered as a ferro-
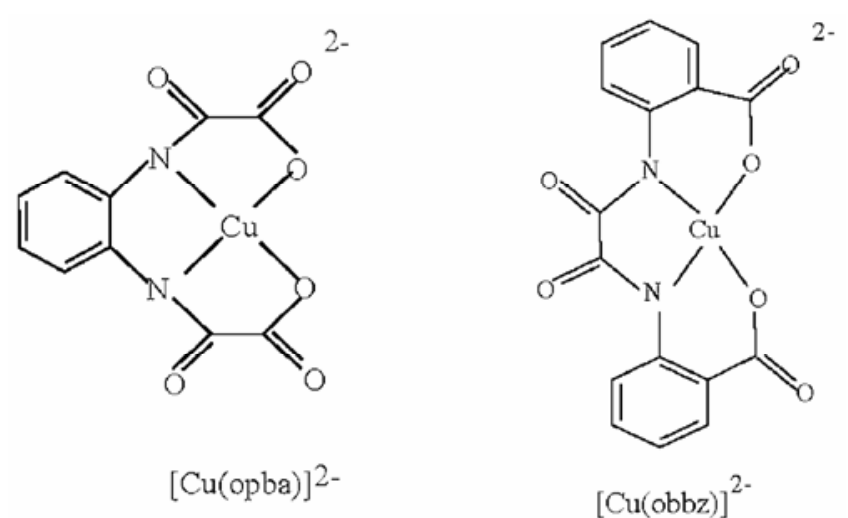

Figure 4. A schematic of the copper dianion precursors $[\mathrm{Cu}(o p b a)]^{2-}$ and $[\mathrm{Cu}(o b b z)]^{2-}$, where 'opba' and 'obbz' stands for ortho-phenylenebis(oxamato) and oxamido bis(benzoato), respectively. 
magnetic coupling of the ground state spin $/ S_{\mathrm{A}}-S_{\mathrm{B}} /$ of the $\mathrm{AB}$ units, which in the case of $\mathrm{Mn}(\mathrm{II}) \mathrm{Cu}(\mathrm{II})$-based compounds is, $S=2$. The compound $\mathrm{MnCu}(o b b z) \cdot 5 \mathrm{H}_{2} \mathrm{O}$ (Nakatani et al 1989), obtained by the polymerization of ferrimagnetic molecular precursor units prepared by reacting the copper dianion $[\mathrm{Cu}(o b b z)]^{2-}$ (figure 4), where $o b b z$ is the ligand oxamido bis(benzoato), with $\mathrm{Mn}(\mathrm{II})$ ions, exhibits a minimum at $44 \mathrm{~K}$ in its $\chi_{\mathrm{M}} T$ versus $T$ plot, $\chi_{\mathrm{M}}$ being the molar magnetic susceptibility and $T$ the temperature, a signature of a one-dimensional ferrimagnet, and a sharp maximum at $2.3 \mathrm{~K}$ due to a threedimensional antiferromagnetic ordering. Upon dehydration, it yields the monohydrate, $\mathrm{MnCu}(o b b z) \cdot 1 \mathrm{H}_{2} \mathrm{O}$, which orders ferromagnetically below the critical temperature $\left(T_{\mathrm{c}}\right)$ of $14 \mathrm{~K}$, due to the non-compensation of the magnetic moments on $\mathrm{Mn}(\mathrm{II})$ and $\mathrm{Cu}(\mathrm{II})$ (Nakatani et al 1989; Chavan et al 1995). Subsequently, Ni(II)-, Fe(II)and $\mathrm{Co}(\mathrm{II})$-based bimetallic chain compounds have also been synthesized using the $[\mathrm{Cu}(o b b z)]^{2-}$ precursor (Nakatani et al 1989; Chavan et al 1995; Larionova et al 1997; Kahn et al 1999; Sra et al 2001). Using the mixed metal ion spin-organic radical spin approach, a $46 \mathrm{~K}$ magnet was synthesized by the reaction of a trinitroxide radical, with three parallel spins $(S=3 / 2)$, with bis(hexafluoroacetylacetonato)Mn(II), [Mn(II)(hfac) $)_{2}$ (Inoue et al 1996).

The Mn(II) ion is not able to prevent the domains from rotating freely under an applied field because it is a magnetically isotropic ion. Most of the $\mathrm{Mn}(\mathrm{II}) \mathrm{Cu}(\mathrm{II})$-based magnets, though exhibit a high value of $T_{\mathrm{c}}(12$ to $30 \mathrm{~K})$, are soft ferromagnets, therefore, exhibiting rather narrow magnetic hysteresis loops below $T_{\mathrm{c}}$ with rather weak coercive field values $\left(H_{\mathrm{c}}<50 \mathrm{Oe}\right.$ at $4.2 \mathrm{~K}$ ) (Stumpf et al 1993a, b, 1994a, b). The same is generally true for $\mathrm{Fe}(\mathrm{II}) \mathrm{Cu}$ (II)-based magnets. It is the coercivity of a magnet which confers a memory effect on it. Stronger coercive fields are expected for $\mathrm{Co}^{2+}$-based molecular magnets where $\mathrm{Co}^{2+}$ ion in distorted octahedral environment, being magnetically anisotropic, can assume preferred orientations. Replacing $\mathrm{Mn}(\mathrm{II})$, with an orbital singlet state $\left({ }^{6} \mathrm{~A}_{1}\right)$, by $\mathrm{Co}(\mathrm{II})$ with an orbital triplet ground state $\left({ }^{4} \mathrm{~T}_{1}\right)$ in (cat) $)_{2} \mathrm{Mn}_{2}[\mathrm{Cu}(o p b a)]_{3}$.S (Stumpf et al 1993a, b, 1994a) resulted in a dramatic rise in coercivity. For instance, $H_{\mathrm{c}}=3000 \mathrm{Oe}$ for $\left(\mathrm{NBu}_{4}\right)_{2} \mathrm{Co}_{2}[\mathrm{Cu}(\text { opba })]_{3} \cdot 3 \mathrm{DMSO} \cdot 3 \mathrm{H}_{2} \mathrm{O}$ and $3100 \mathrm{Oe}$ for $(\mathrm{rad})_{2} \mathrm{Co}_{2}[\mathrm{Cu}(o p b a)]_{3} \cdot 0 \cdot 5 \mathrm{DMSO} \cdot 3 \mathrm{H}_{2} \mathrm{O}$ (Stumpf et al 1994b), where 'rad' stands for the radical cation 2-(4-N-methylpyridinium)-4,4,5,5-tetramethylimidazolin-1-oxyl 3-oxide and 'opba' denotes orthophenylene-bis(oxamato). $\quad\left[(\text { Etrad })_{2} \mathrm{Co}_{2}\{\mathrm{Cu}(\text { opba })\}_{3}\right.$ (DMSO $\left.)_{1.5}\right] \cdot 0 \cdot 25 \mathrm{H}_{2} \mathrm{O}$ exhibits high coercivity, up to $24 \mathrm{kOe}$ at $6 \mathrm{~K}$ (Vaz et al 1999). These values are much higher than that for the commercial atom-based materials, $\mathrm{Fe}_{2} \mathrm{O}_{3}$ or $\mathrm{CrO}_{2}$. The synthetic procedures employed to obtain molecular materials are different from those employed in solid state chemistry and the molecular crystal lattice is characteristically soft, as compared to the ionic/metallic lattices. We have exploited these attributes to demonstrate that for certain molecular magnets, assembled from $\mathrm{Co}$ (II) $\mathrm{Cu}$ (II) based ferrimagnetic chains, it is possible to modify the magnetic properties dramatically and reversibly through a mild dehydrationrehydration process, and have named this class of compounds as molecular magnetic sponges (Turner et al 1996; Larionova et al 1997; Chavan et al 1998; Kahn et al 1999). This is because they show 'sponge'-like characteristics, viz. a reversible cross-over under dehydration to a polymerized long range magnetically ordered state with spontaneous magnetization, and transforming back into the isolated units underlying the initial non-magnetic phase by reabsorbing water, i.e. re-hydration of both noncoordinated and coordinated water molecules (figure 5). Coercivity values for these sponges are high and for some of them a colour change, too, occurs reversibly and simultaneously with the change in magnetic properties at the transition temperature corresponding to the dehydration-rehydration process. In the case of $\mathrm{CoCu}(o b b z)$. $n \mathrm{H}_{2} \mathrm{O}$, we confirmed that the $\mathrm{Co}-\mathrm{O}$ bonds could be broken and created without destroying the essence of the molecular architecture. The main features of the four $\mathrm{Co}(\mathrm{II}) \mathrm{Cu}(\mathrm{II})$-based molecular magnetic sponges synthesized by our group, viz. $\mathrm{CoCu}(p b a \mathrm{OH})\left(\mathrm{H}_{2} \mathrm{O}\right)_{3} \cdot 2 \mathrm{H}_{2} \mathrm{O}$, $\mathrm{CoCu}(p b a)\left(\mathrm{H}_{2} \mathrm{O}\right)_{3} \cdot 2 \mathrm{H}_{2} \mathrm{O}, \quad \mathrm{CoCu}(o b b z)\left(\mathrm{H}_{2} \mathrm{O}\right)_{4} \cdot 2 \mathrm{H}_{2} \mathrm{O}$ and $\mathrm{CoCu}($ obze $)\left(\mathrm{H}_{2} \mathrm{O}\right)_{4} \cdot 2 \mathrm{H}_{2} \mathrm{O}$ are high values of $T_{\mathrm{c}}(38,33,25$ and $25 \mathrm{~K}$, respectively) and $H_{\mathrm{c}}(5.66,3,1.3$ and $1 \mathrm{kOe}$, respectively).

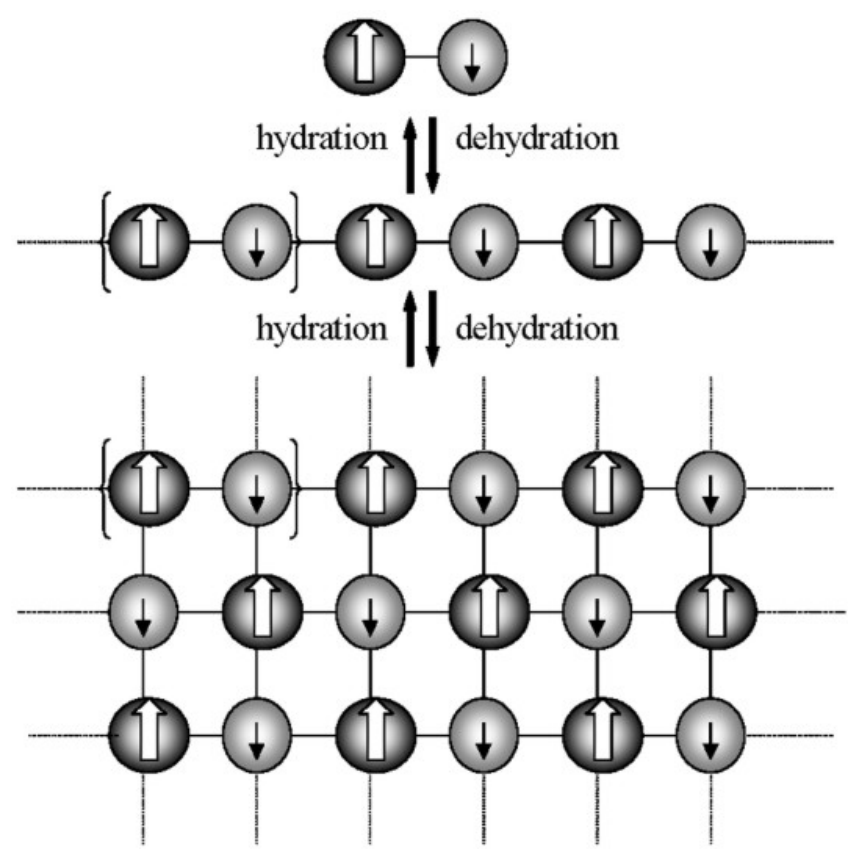

Figure 5. The dehydration-polymerization process, typically applicable to the reversible sponge-like behaviour of the molecular magnet, $\mathrm{CoCu}(o b b z) \cdot n \mathrm{H}_{2} \mathrm{O}$. Reproduced from Kahn et al (1999) with permission from Wiley-VCH Verlag GmbH \& Co. KgaA (copyright). 


\section{Polycyanometallates}

A unique feature of the molecular magnets is that they are usually weakly coloured unlike the opaque classical magnets. Design of molecular ferromagnets of low density that are transparent and have a tunable high $T_{\mathrm{c}}$ is a cherished goal and photomagnetic switching has been reported in molecular magnets, especially where hexacyanometalates, $\left[\mathrm{M}(\mathrm{CN})_{6}\right]^{n-}$, are used as molecular building blocks. Most metal hexacyanometallates have a cubic $(f c c)$ structure and are analogues of the well-known Prussian Blue salt, $\quad \mathrm{Fe}^{\mathrm{III}}{ }_{4}\left[\mathrm{Fe}^{\mathrm{II}}(\mathrm{CN})_{6}\right]_{3} \cdot 15 \mathrm{H}_{2} \mathrm{O}$, whose structure was described by Ludi and Gudel (1973) as highly disordered cubic cell consisting of alternating ferrocyanide and ferric ions, with linear $\mathrm{Fe}^{\mathrm{III}}-\mathrm{N}-\mathrm{C}-\mathrm{Fe}^{\mathrm{II}}$ bridges. In the case of Prussian Blue analogues, $\mathrm{A}_{\mathrm{k}}\left[\mathrm{B}(\mathrm{CN})_{6}\right] \cdot x \mathrm{H}_{2} \mathrm{O}$, the transition metal ions, $\mathrm{A}$ and $\mathrm{B}$, are situated at the corners of the cube (figure 6), and the cyanide groups bridge the metal ions along the cube edges, the metal ions being octahedrally coordinated by the nitrogen or carbon end of the cyanide group. Depending on the charged-state of the $\mathrm{N}$ - and C-coordinated metal ions, a certain number of cations can occupy interstitial positions for charge compensation. These interstitial metal ions may be the same as the $\mathrm{N}$ - and $\mathrm{C}$-coordinated metal ions, or they may be alkali metal ions.

The cyano bridge is known to mediate strong antiferromagnetic or ferromagnetic interactions (Entley and Girolami 1995). $T_{\mathrm{c}}$ values above room temperatures, viz. $376 \mathrm{~K}$ (Holmes and Girolami 1999), have been reported for hexacyanometalates but their high $f c c$ symmetry is often accompanied by inherent disorder among different

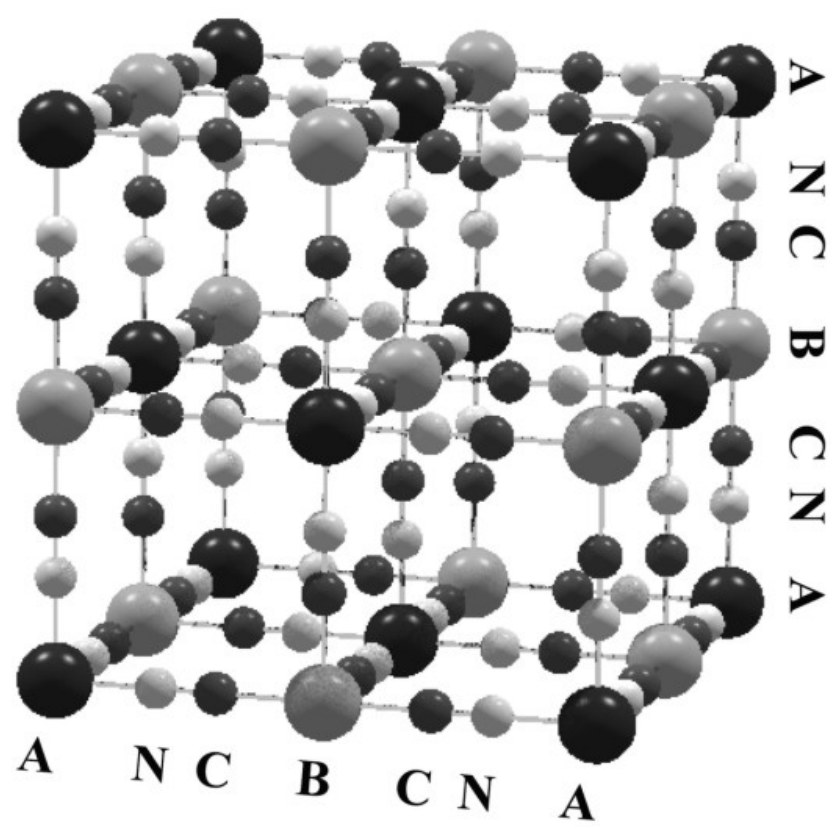

Figure 6. $F C C$ structure of Prussian Blue analogues, $\mathrm{A}\left[\mathrm{B}(\mathrm{CN})_{6}\right] \cdot z \mathrm{H}_{2} \mathrm{O}$. For clarity, $\mathrm{H}$ and $\mathrm{O}$ atoms are not shown. cationic sites, making it difficult to grow single crystals or to study any magnetic anisotropy. In the PBAs, $\mathrm{A}_{\mathrm{m}}\left[\mathrm{B}(\mathrm{CN})_{6}\right]_{n} \cdot z \mathrm{H}_{2} \mathrm{O}$ with $m>n$, the $\left[\mathrm{B}(\mathrm{CN})_{6}\right]$ vacancies, which maintain the required charge neutrality, and partial occupancies of the non-coordinated water molecules constitute the main structural defects. As the magnetic properties of a compound depend on its crystal structure, the study of defects and disorders ought to be carried out systematically for proper understanding of the possible correlation between the structural defects and magnetic ordering. Substitution of $\mathrm{Ru}$ at Ni-site introduces a substitutional disorder in $\mathrm{Ru}_{x} \mathrm{Ni}_{3-3 x / 2}\left[\mathrm{Cr}(\mathrm{CN})_{6}\right]_{2} \cdot z \mathrm{H}_{2} \mathrm{O}$ compounds. The effect of $\mathrm{Ru}^{3+}$ ion substitution on the structural defects/disorders in this system has been studied by us (Kumar et al 2007) employing X-ray diffraction, IR spectroscopy, d.c. magnetization and neutron diffraction. The influence on $T_{\mathrm{C}}$ with increasing substitution of $\mathrm{Ru}$ can be explained on the extent of the structural disorder, and this disorder itself was estimated by bus from the Reverse Monte Carlo treatment of the observed neutron diffraction patterns.

We have demonstrated that one can grow crystals of Prussian Blue Analogues (NiHCF, CoHCF) at the airwater interface using a surfactant (octadecylamine) monolayer as a template (Choudhury et al 2002, 2003; Bagkar et al 2006a). The AFM image of the crystalline film of cobalt-hexacyanoferrate grown over three days showed a granular structure (grain size, $\sim 200 \mathrm{~nm}$ ). SQUID magnetometry on nanocrystals of nickel hexacyanoferrate (Nihcf) transferred as a monolayer showed that they were magnetic below $20 \mathrm{~K}$. Sodium hexametaphosphate (HMP) stabilized nickel hexacyanoferrate

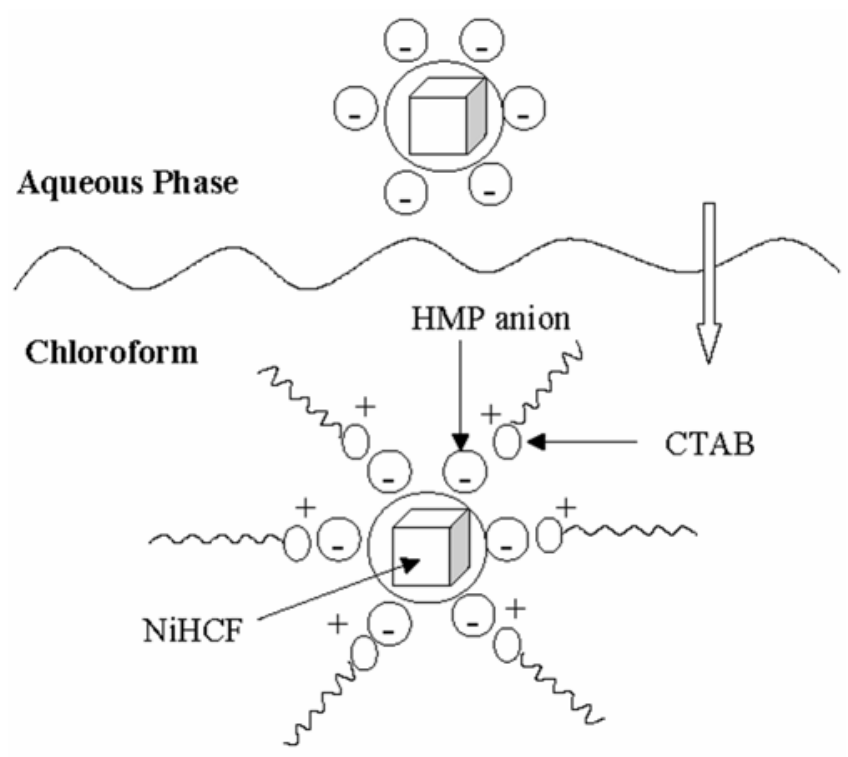

Figure 7. Schematic representation of extraction of NiHCF nanoparticles from the aqueous phase to the organic phase. Reproduced from Bagkar et al (2004), with permission of The Royal Society of Chemistry (copyright) 
(NiHCF) nanoparticles were prepared in aqueous solution and were successfully extracted into an organic phase (figure 7) using cetyltrimethylammonium bromide (CTAB) as the surfactant (Bagkar et al 2004). Potassium ion sensing action was demonstrated by us by employing cyclic voltammetry and impedance measurements on NiHCFDODA films (DODA: dioctadecyl dimethylammonium bromide) (Bagkar et al 2006b). We have also used the double-stranded CT-DNA as a template to self-assemble crystals of nickel hexacyanoferrate, $\mathrm{Ni}_{3}\left[\mathrm{Fe}(\mathrm{CN})_{6}\right]_{2}$. $14 \mathrm{H}_{2} \mathrm{O}$, with an average diameter of $400 \mathrm{~nm}$. As a function of temperature, the morphology of NiHCF crystals can change and they self-assemble into linear ropes, spheres, and dendritic structures (Bagkar et al 2008).

An increase in the surface to volume ratio, due to a reduction in particle size, and inter-particle interactions play an important role in deciding the magnetic properties of nanoparticle systems. We have compared the observed magnetic behaviour of $\mathrm{Fe}\left[\mathrm{Fe}(\mathrm{CN})_{6} \cdot x \mathrm{H}_{2} \mathrm{O}\right.$ nanoparticles (mean diameter $\sim 50 \mathrm{~nm}$ ) prepared using a water-in-oil microemulsion technique, with that of its bulk counterpart. A magnetic ordering temperature, $T_{\mathrm{C}}$ of $13.0 \mathrm{~K}$ was estimated from the magnetization data, which is low compared to that for the bulk polycrystalline sample, $T_{\mathrm{C}}=17.4 \mathrm{~K}$, and suggests the presence of a weak surface spin disorder in the ferromagnetically ordered cores of nanoparticles, the surface spin-disorder causing a spin glass behaviour (Mukadam et al 2008).

\subsection{Negative magnetization and pole-reversal}

A crossover of the field-cooled magnetization from positive to negative below the magnetic ordering temperature is a unique feature exhibited by molecular multi-cation ferrimagnets (Chavan et al 1996; Mathoniere et al 1996); and by Prussian Blue Analogues (Ohkoshi et al 1999). We have recently provided the first neutron magnetic structure evidence towards the microscopic understanding of the negative magnetization phenomenon in the PBA, $\mathrm{Cu}_{0.73} \mathrm{Mn}_{0.77}\left[\mathrm{Fe}(\mathrm{CN})_{6}\right] \cdot z \mathrm{H}_{2} \mathrm{O} \quad\left(T_{\mathrm{c}}=17.9 \mathrm{~K}\right)$. The Reverse Monte Carlo analysis, combined with the Rietveld refinement technique shows an antiferromagnetic ordering of $\mathrm{Mn}$ moments with respect to the $\mathrm{Cu}$ as well as the $\mathrm{Fe}$ moments (Kumar et al 2008). Apart from temperaturedependent pole-reversal it also exhibited reversible (bipolar) switching of magnetization under low magnetic fields, which leads to the reversal of magnetic entropy change $\left(\Delta S_{\mathrm{m}}\right)$, causing a bipolar magneto-caloric effect (MCE), i.e. both positive and negative MCEs below the magnetic ordering temperature. These novel phenomena related to pole reversal could find applications in novel devices such as magnetic memory, thermomagnetic switches, and magnetic cooling/heating based constant temperature baths.

Apart from the fact that a molecule is the ultimate unit for data-storage, the design of molecular magnets has also opened the doors for the unique possibility of designing poly-functional materials, at the molecular level such as those exhibiting ferromagnetic behaviour and second-order optical nonlinearity (Benard et al 2000), or, liquid crystallinity and magnetism (Binnemans et al 2000). In collaboration with Prof. Inoue's group from Japan, we designed a three-dimensional chiral, transparent ferrimagnet, $\mathrm{K}_{0.4}\left[\mathrm{Cr}(\mathrm{CN})_{6}\right][\mathrm{Mn}(S)-p n](S)-p n \mathrm{H}_{0.6}$ with $T_{\mathrm{c}}=53 \mathrm{~K}$, where $(S)-p n$ stands for $(S)$-1,2-diaminopropane) (Inoue et al 2001), and there have been many additions to this class of bi-functional magnets, since.

\subsection{Hepta/octacyanates}

An alternate strategy adopted by us to grow single crystals of cyanometallates was to employ the heptacyanate anion $\left[\mathrm{Mo}^{\mathrm{III}}(\mathrm{CN})_{7}\right]^{4-}$ as a precursor because its pentagonal bipyramidal coordination sphere is incompatible with a cubic lattice. Reaction of $\mathrm{Mn}^{\mathrm{II}}$ ions with the heptacyanate $\left[\mathrm{Mo}^{\mathrm{III}}(\mathrm{CN})_{7}\right]^{4-}$ precursor led to lowering of symmetry and high values of Curie temperatures $\left(T_{\mathrm{c}}=51 \mathrm{~K}\right)$ arising from a ferromagnetic interaction between the low spin $\mathrm{Mo}^{3+}$ and the high spin $\mathrm{Mn}^{2+}$ through the $\mathrm{Mo}^{\mathrm{III}}-\mathrm{C}-$ $\mathrm{N}-\mathrm{Mn}^{\mathrm{II}}$ bridges. The use of a macrocycle reduced the symmetry further by imposing hepta-coordination on $\mathrm{Mn}^{\mathrm{II}}$ ion, too, but a low $T_{\mathrm{c}}(3 \mathrm{~K})$ for the compound $\left[\mathrm{Mn}^{\mathrm{II}} \mathrm{L}\right]_{6}\left[\mathrm{Mo}^{\mathrm{III}}(\mathrm{CN})_{7}\right]\left[\mathrm{Mo}^{\mathrm{IV}}(\mathrm{CN})_{8}\right]_{2} \cdot 19 \cdot 5 \mathrm{H}_{2} \mathrm{O}$, where $L$ is the macrocycle (figure 8), which was attributed to the existence of diamagnetic $\mathrm{Mo}^{4+}$ along certain - $\mathrm{CN}$ - bridges, in addition to the paramagnetic $\mathrm{Mo}^{3+}$ (Sra et al 1999). We also demonstrated the use of octacyanometalates as versatile building blocks (Sra et al 2000). Ohkoshi et al (2006) reported reversible switching between paramagnetic and ferromagnetic states for $\mathrm{Cu}_{2}^{\mathrm{II}}\left[\mathrm{Mo}^{\mathrm{IV}}(\mathrm{CN})_{8}\right] \cdot 8 \mathrm{H}_{2} \mathrm{O}$ solid by irradiating it with visible lights, which is very encouraging. However, its $T_{\mathrm{c}}$ is low $(25 \mathrm{~K})$, and to be practically useful, the $T_{\mathrm{c}}$ value in the extended network must be raised to above room temperature.

\section{Open-framework magnetic structures}

Magnetic zeolitic structures offer excellent conditions to encapsulate different functional systems with conducting, optical, chiral and NLO properties, among others. It is desirable, therefore, to evolve a synergism of magnetic and nanoporous behaviour, together with the molecular characteristics of coordination polymers, as a new route to the development of multifunctional molecular materials. Veciana and co-workers (Maspoch et al 2003, 2004) have fabricated a nanoporous molecular magnet, $\mathrm{Cu}_{3}(\mathrm{PTMTC})_{2}$ $(p y)_{6}\left(\mathrm{CH}_{3} \mathrm{CH}_{2} \mathrm{OH}\right)_{2}\left(\mathrm{H}_{2} \mathrm{O}\right)$, [PTMTC being a radical, viz. polychlorinated triphenylmethyl with three carboxylic groups] with reversible solvent-induced mechanical and magnetic properties. This compound has an openframework structure, with very large pores $(2 \cdot 8-3 \cdot 1 \mathrm{~nm})$ 


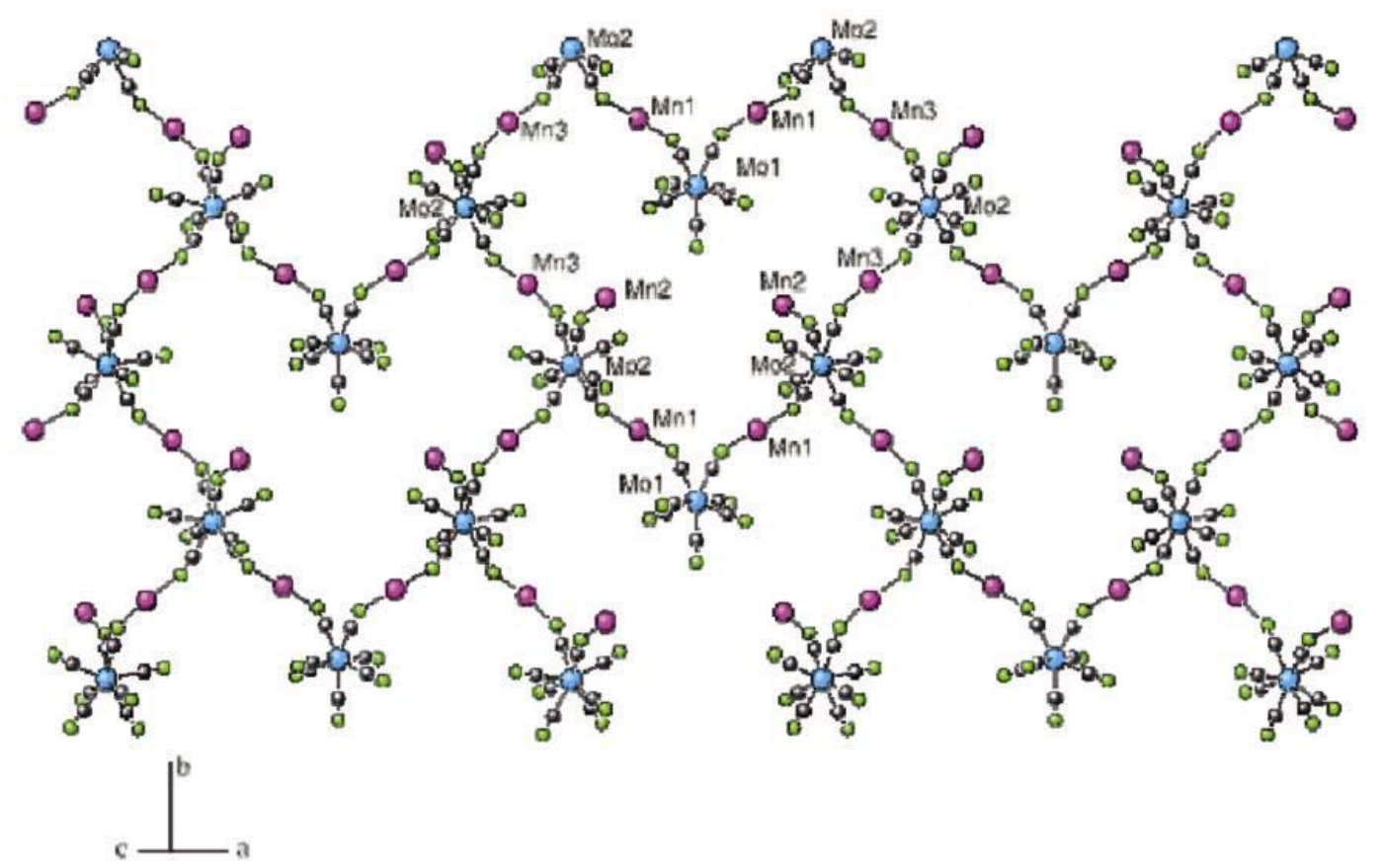

Figure 8. The compound $\left[\mathrm{Mn}^{\mathrm{II}} \mathrm{L}\right]_{6}\left[\mathrm{Mo}^{\mathrm{III}}(\mathrm{CN})_{7}\right]\left[\mathrm{Mo}^{\mathrm{IV}}(\mathrm{CN})_{8}\right]_{2} \cdot 19 \cdot 5 \mathrm{H}_{2} \mathrm{O}$, where $\mathrm{L}$ is a macrocycle. Reproduced from Sra et al (1999), with permission from Wiley-VCH Verlag GmbH \& Co. KgaA (copyright).

and bulk magnetic ordering. Reversible behaviour has only been observed for ethanol and methanol, showing the selectivity of this sponge-like magnetic sensor.

\section{Single molecule magnets}

Synthesis activity in molecular magnets has recently led to the development of what are known as single molecule magnets (SMMs), such as $\mathrm{Mn}_{12} \mathrm{O}_{12}\left(\mathrm{CH}_{3} \mathrm{COO}\right)_{16}\left(\mathrm{H}_{2} \mathrm{O}\right)_{4}$, with large spin ground state (e.g. $S=10$ ). Crystals of SMMs function as collections of monodisperse nanomagnets, unlike other nanoscale magnetic materials which have size distribution. As magnetic aggregates, the SMMs are examples of 'zero-dimensional' systems, though essentially mesoscopic, and therefore, lie on the border between the classical and quantum regimes, serving as model systems to study new sources of magnetic phenomena including quantum tunneling, quantum coherence, QuBits, magnetocaloric effect, etc (Friedman et al 1996; Barbara and Gunther 1999; Leuenberger and Loss 2001). More recently, there is much interest also in what is known as single-chain magnets (SCM), which are ferroor ferrimagnetic chains, and mostly Ising systems. A typical example of SCMs is the cobalt(II)-organic radical polymer $\mathrm{Co}(h f a c)_{2} \mathrm{NITPhOMe}$, nicknamed 'CoPhOMe' (Bogani et al 2005). Relaxation in these isotropic ferromagnetic chains follow Glauber's dynamics, and no quantum tunneling is observed, unlike in SMMs. We have, however, studied slow magnetic relaxations in $\mathrm{Mn}$ (III) tetra(ortho-fluorophenyl)porphyrin-tetracyanoethylene, an anisotropic Heisenberg chain compound consisting of ferrimagnetic chains, in which magnetic moments of $\mathrm{Mn}$ ion $(S=2)$ in the centre of a porphyrin disc and of a tetracyanoethylene radical $(s=1 / 2)$, are antiferromagnetically coupled (Bałanda et al 2006).

\section{Current scenario and future perspectives}

Magnets fabricated from molecules are unique to magnetism. They exhibit all the phenomena observed in conventional transition-metal and rare earth-based magnets, and more, by exhibiting liquid crystallinity, chirality, low density, solubility, spin-crossover (Krober et al 1993), negative magnetization (Mathoniere et al 1994; Chavan et al 1996; Kumar et al 2008), photoinduced magnetization/switching due to their coloured nature (Hashimoto and Ohkoshi 1999; Sastry et al 1999; Pejakovic et al 2002), and possibility of modulation of their properties electrochemically, all of which are arising from their characteristic molecular nature. In this context, an exciting development is the fabrication of a tri-functional phenalenyl-based neutral radical, by Itkis et al (2002), which exhibits magneto-opto-electronic bistability with hysteresis loops centred near $335 \mathrm{~K}$, thus opening the possibility for new type of electronic devices, where multiple physical channels can be used for writing, reading, and transferring information.

Some groups are attempting to utilize the high degree of directionality of hydrogen bonding between open-shell 
molecules to obtain supramolecular self-organization aimed at achieving new molecular ferromagnets. There is also a substantial activity and progress in the development of spin-crossover compounds that exhibit magnetic bistability (Krober et al 1993; Fujita and Awaga 1999), which is at times accompanied by a change of colour, too. Then, there is a continuing quest for discovering new molecular materials which can exhibit long range magnetic order at room temperature (or even above), the earliest example of which was V[TCNE $]_{x} \cdot y \mathrm{CH}_{2} \mathrm{Cl}_{2} \quad(x \sim 2$; $y \sim 1 / 2$ ), a disordered, amorphous magnet depicting a $T_{\mathrm{c}}$ of $\sim 400 \mathrm{~K}$, but extremely water/air sensitive (Zhang et al 1996). Jain et al (2007) recently reported the synthesis of three air-stable $\mathrm{Ni}_{2} \mathrm{~A}$-based metal-organic magnets $\left(T_{\mathrm{c}}\right.$ above room temperature), by reacting a metal $(\mathrm{M})$ precursor complex bis(1,5-cyclooctadiene)nickel with organics $\mathrm{A}=\mathrm{TCNE}$ (tetracyanoethylene), TCNQ (7,7,8,8-tetracyanoquinodimethane) or DDQ (2,3-dichloro-5,6-dicyano1,4-benzoquinone). A structural model for these materials is not available, though spectroscopic features suggested that the organic ligands are $\sigma$-bound to nickel. However, subsequent study by Miller and Pokhodnya (2007) has negated these findings, and proposed that the black powdery magnetic material made upon dissolution of $\mathrm{Ni}(\mathrm{COD})_{2}$ in $\mathrm{CH}_{2} \mathrm{Cl}_{2}$ presumably consists of 'nano'- or bigger-sized particles of nickel metal, and lacks the 'organic' species which may entitle it to be called a molecular magnet.

Unique combination of tunability, processability, and other attributes of molecular lattices hold promise for molecule-based magnets for future applications, but much progress has to be made before that happens because of certain vexing issues like their air stability, degradability (in some cases) and processability, phenomena unique to molecular lattices. Perhaps, one has to learn more from the self-assembly processes of Nature, which synthesizes monodisperse nano-scale single-domain magnetite crystals in magnetotactic bacteria, which collectively behave as bar magnets, imparting the bacteria an ability to orient and migrate along geomagnetic field lines. These nanocrystals form in intracellular magnetic structures, called magnetosomes, via a biological mechanism that controls their biomineralization (Philipse and Mass 2002). Biogenic magnetite has also been detected in animals (honeybees, birds, salmon, newts, sea turtles, spiny lobsters etc), which use them to transduce geomagnetic field information to the nervous system for 'homing' action (Lohmann and Johnsen 2000).

\section{Acknowledgements}

This article is dedicated to Prof. C N R Rao on his 75th birthday. The intensity and enthusiasm of Prof. Rao in pursuing different aspects of the science of materials has been a great source of inspiration. In the area of molecu- lar magnetism, the author has benefitted enormously by his collaborations with the late Prof. Olivier Kahn, a pioneer in the area of molecular magnetism. The author has also benefitted from interaction with Prof. K Inoue and Dr M D Sastry, and would, in addition like to place on record his deep appreciation for the contributions of his colleagues Drs S M Yusuf, Sipra Choudhury, G K Dey, M D Mukadam and past and present students S A Chavan, Aman K Sra, Prasanna Ghalsasi, Nitin Bagkar, Nidhi Sharma, Amit Kumar on which this article is largely based. Help extended by Dr S M Yusuf in the preparation of some figures is acknowledged.

\section{References}

Allemand P, Khemani K, Koch K, Wudl F, Holczer K, Donovan S, Gruner G and Thompson J D 1991 Science 253301

Arita R, Suwa Y, Kuroki K and Aoki H 2002 Phys. Rev. Lett. 88127202

Bagkar N, Ganguly R, Choudhury S, Hassan P A and Yakhmi J V 2004 J. Mater. Chem. 141430

Bagkar N, Choudhury S, Kim K H, Chowdhury P, Lee S I and Yakhmi J V 2006a Thin Solid Films 513325

Bagkar N, Betty C A, Hassan P A, Kahali K, Bellare J R and Yakhmi J V 2006b Thin Solid Films 497259

Bagkar N, Choudhury S, Bhattacharya S and Yakhmi J V 2008 J. Phys. Chem. Part C 1126467

Bałanda M, Rams M, Nayak S K, Tomkowicz Z, Haase W, Tomala K and Yakhmi J V 2006 Phys. Rev. B74 224421

Banister A J et al 1996 Angew. Chem. Int. Ed. 352533

Barbara B and Gunther L 1999 Physics World 35

Benard S, Yu P, Audiere J P, Riviere E, Clement R, Guilhem J, Tchertanov L and Nakatani K $2000 \mathrm{~J}$. Am. Chem. Soc. 122 9444

Binnemans K et al 2000 J. Am. Chem. Soc. 1224335

Bogani L et al 2005 Phys. Rev. B72 064406

Broderick W E, Thompson J A, Day P and Hoffman B M 1990 Science 249410

Caneschi A, Gatteschi D, Sessoli R and Rey P 1989 Acc. Chem. Res. 22392

Chavan S A, Yakhmi J V and Gopalakrishnan I K 1995 Mol. Cryst. Liq. Cryst. 27411

Chavan S A, Ganguly R, Jain V K and Yakhmi J V 1996 J. Appl. Phys. 795260

Chavan S A, Larionova J, Kahn O and Yakhmi J V 1998 Philos. Mag. B6 1657

Choudhury S, Bagkar N, Dey G K, Subramanian H and Yakhmi J V 2002 Langmuir 187409

Choudhury S, Dey G K and Yakhmi J V 2003 J. Cryst. Growth 258197

Entley W R and Girolami G S 1995 Science 268397

Friedman J R, Sarachik M P, Tejada J and Ziolo R 1996 Phys. Rev. Lett. 763830

Fujita W and Awaga K 1999 Science 286261

Hashimoto K and Ohkoshi S 1999 Philos. Trans. R. Soc. London $\mathbf{A 3 5 7} 2977$

Holmes S M and Girolami G S 1999 J. Am. Chem. Soc. 121 5593

Inoue K and Iwamura H 1994 J. Am. Chem. Soc. 1163173 
Inoue K, Hayamizu T, Iwamura H, Hashizume D and Ohashi Y 1996 J. Am. Chem. Soc. 1181803

Inoue $\mathrm{K}$, Imai $\mathrm{H}$, Ghalsasi P S, Ohba M, Okawa $\mathrm{H}$ and Yakhmi J V 2001 Angew. Chem. Int. Ed. 404242

Itkis M E, Chi X, Cordes A W and Haddon R C 2002 Science 2961443

Itoh K and Kinoshita M (eds) 2000 Molecular magnetism (Amsterdam: Gordon and Breach Science Publishers)

Jain R, Kabir K, Gilroy J B, Mitchell K A R, Wong K C and Hicks R G 2007 Nature 445291

Kahn O 1993 Molecular magnetism (New York: VCH)

Kahn O 1995 Adv. Inorg. Chem. 43179

Kahn O (ed.) 1996 Magnetism: A supramolecular function, NATO ASI Series C (Dordrecht: Kluwer) Vol. 484

Kahn O, Pei Y, Verdaguer M, Renard J P and Sletten J 1988 J. Am. Chem. Soc. 1117823

Kahn O, Larionova J and Yakhmi J V 1999 Chem. Eur. J. 5 3443

Krober J, Codjovi E, Kahn O, Groliere F and Jay C $1993 \mathrm{~J} . \mathrm{Am}$. Chem. Soc. 1159810

Kumar A, Yusuf S M, Keller L, Yakhmi J V, Srivastava J K and Paulose P L 2007 Phys. Rev. B75 224419

Kumar A, Yusuf S M, Keller L and Yakhmi J V 2008 Phys. Rev. Lett. 101207206

Larionova J, Chavan S A, Yakhmi J V, Froystein A G, Sletten J, Sourisseau C and Kahn O 1997 Inorg. Chem. 366374

Leuenberger M N and Loss D 2001 Nature 410789

Lohmann K J and Johnsen S 2000 Trends in Neuro Sci. 23153

Ludi A and Gudel H U 1973 Struct. Bonding (Berlin) 141

Maspoch D et al 2003 Nature Mater. 2190

Maspoch D, Domingo N, Ruiz-Molina D, Wurst K, Vaughan G, Tejada J, Rovira C and Veciana J 2004 Angew. Chem. Int. Ed. 431828

Mathoniere C, Carling S G, Yusheng D and Day P $1994 J$. Chem. Soc. Chem. Commun. 1551

Mathoniere C, Nuttal C J, Carling S G and Day P 1996 Inorg. Chem. 351201

McConnell H M 1963 J. Chem. Phys. 391910

Miller J S 2000 Inorg. Chem. 394392

Miller J S, Calabrese J C, Epstein A J, Bigelow R B, Zang J H and Reiff W M 1986 J. Chem. Soc.: Chem. Commun. 1026

Miller J S and Pokhodnya K I 2007 J. Mater. Chem. 173585

Mito M, Tanimoto T, Kawae T, Hitaka M, Takeda K, Nakatsuji S, Morimoto H and Anzai H 2001 Polyhedron 201509

Mrzel A, Omerzu A, Umek P, Mihailovic D, Jaglicic Z and Trontelj Z 1998 Chem. Phys. Lett. 298329

Mukadam M D, Kumar K, Yusuf S M, Yakhmi J V, Tewari R and Dey G K 2008 J. Appl. Phys. 103123902
Nakatani K et al 1989 J. Am. Chem. Soc. 1115739

Neels A, Stoeckli-Evans H, Chavan S A and Yakhmi J V 2001 Inorg. Chim. Acta 326106

Okawa H, Mitsumi M, Ohba M, Kodera M and Matsumoto N 1994 Bull. Chem. Soc. Jap. 672139

Ohkoshi S I, Abe Y, Fujishima A and Hashimoto K 1999 Phys. Rev. Lett. 821285

Ohkoshi S I et al 2006 J. Am. Chem. Soc. 128270

Ovchinnikov A A 1978 Theor. Chim. Acta 47297

Palacio F, Antorrena G, Castro M, Burriel R, Rawson J, Smith J N, Bricklebank N, Novoa J and Clemens R 1997 Phys. Rev. Lett. 792336

Pei Y, Verdaguer M, Kahn O, Sletten J and Renard J P 1986 J. Am. Chem. Soc. 108428

Pei Y, Kahn O, Nakatani K, Codjovi E, Mathoniere C and Sletten J 1991 J. Am. Chem. Soc. 1136558

Pejakovic D A, Kitamura C, Miller J S and Epstein A E 2002 Phys. Rev. Lett. 88057202

Philipse A P and Mass D 2002 Langmuir 189977

Rajca S, Wongsriratanakul J and Rajca S 2001 Science 294 1503

Sastry M D, Bhide M K, Kadam R M, Chavan S A, Yakhmi J V and Kahn O 1999 Chem. Phys. Lett. 301385

Sra A K, Andruh M, Kahn O, Golhen S, Ouahab L and Yakhmi J V 1999 Angew. Chem. Int. Ed. 382606

Sra A K, Rombaut G, Lahitete F, Golhen S, Ouahab L, Mathoniere C, Yakhmi J V and Kahn O 2000 New J. Chem. 24871

Sra A K, Tokumoto M and Yakhmi J V 2001 Philos. Mag. B81 477

Stumpf H O, Pei Y, Kahn O, Sletten J and Renard J P 1993a J. Am. Chem. Soc. 1156738

Stumpf H O, Ouahab L, Pei Y, Grandjean D and Kahn O 1993b Science 261447

Stumpf H O, Ouahab L, Pei Y, Bergerat P and Kahn O 1994a J. Am. Chem. Soc. 1163866

Stumpf H O, Pei Y, Michaut C, Kahn O, Renard J P and Ouahab L 1994b Chem. Mater. 6257

Tamura M, Nakazawa Y, Shiomi D, Nozawa K, Hosokoshi Y, Ishikawa M, Takahashi M and Kinoshita M 1991 Chem. Phys. Lett. 186401

Turner S, Kahn O and Rabardel L 1996 J. Am. Chem. Soc. 118 6428

Vaz M G F et al 1999 Chem. Eur. J. 51486

Yakhmi J V 2003 Proc. of INAE conf. on nanotechnology (ICON-2003) (ed.) M J Zarabi (Indian National Acad. of Engineering) pp 78-87

Zhang J, Zhou P, Brinckerhoff W B, Epstein A J, Vazquez C, McLean R S and Miller J S 1996 A. C. S. Symp. Ser. 644311 